\title{
Focused abdominal ultrasound in preoperative liver surgery staging: a prospective study
}

\author{
Marcela P Cohen ${ }^{1 *}$, Paulo Hermann ${ }^{2}$, Rubens Chojniak', Miriam RB Poli', Paula NV Barbosa ${ }^{1}$ \\ and Almir GV Bitencourt ${ }^{1}$
}

\begin{abstract}
Background: Because of its safety, relative low cost and widespread availability, conventional ultrasound (US) is the modality of choice for initial evaluation of the liver. Following US, in patients eligible for surgery, further computed tomography and/or magnetic resonance imaging is usually recommended for surgical planning. There are no recent published series focusing on conventional abdominal US exclusively employed for the evaluation of liver nodules before surgery. The objective of this study is to evaluate the efficacy of focused conventional preoperative US in detecting liver lesions, and the impact of US findings on surgical management.
\end{abstract}

Methods: Sixty-seven noncirrhotic patients underwent surgical resection, after being previously submitted to focused liver US evaluation. US results were compared with intraoperative US (IOUS) and histology (gold standard). The IOUS was performed by the same radiologist who performed the preoperative US. Patient-by-patient and lesion-by-lesion analyses were performed.

Results: A total of 241 lesions were depicted in 67 patients. The mean number of lesions detected per patient by US and IOUS was 2.37 and 3.37, respectively $(P=0.001)$. In $52.2 \%$ of patients, US and IOUS depicted the same number of liver lesions. Surgery with curative intent was conducted in 61 (91.0\%) patients. Histological evaluation was obtained in 196 lesions; 155 were considered malignant. The overall lesions detection rate by US was 65.6\%. For lesions $<15 \mathrm{~mm}$ and lesions $\geq 15 \mathrm{~mm}$, US showed a sensitivity rate of $55.3 \%$ and $75.5 \%$, respectively.

Conclusions: The relatively high sensitivity rates achieved by US focused on liver evaluation, with the aim of lowering costs but not efficiency, places the method in focus again for use in the routine preoperative staging of candidates for liver resection. We suggest for preoperative evaluation that US could be associated with one section imaging method (computed tomography or magnetic resonance imaging) as routine.

Keywords: Ultrasonography, Liver neoplasms, Preoperative care

\section{Background}

Advances in imaging diagnosis, tumor staging, preoperative and postoperative care and surgical techniques have had a great impact on liver metastases resection in the last years. This impact is particularly evident in patients with colorectal cancer and neuroendocrine tumor metastases, and in selected cases with other neoplasms [1-3].

Higher survival rates are related to complete removal of hepatic metastases, and the success of the surgical treatment highly depends on preoperative staging [4]. Several

\footnotetext{
* Correspondence: ecmpnm@terra.com.br

'Department of Imaging, AC Camargo Cancer Center, Rua Professor Antônio Prudente, 211, São Paulo, Brazil

Full list of author information is available at the end of the article
}

imaging techniques are employed for preoperative staging of the liver and, in clinical practice, ultrasound (US), computed tomography (CT) and magnetic resonance imaging (MRI) are currently used for that purpose.

Because of its safety, relative low cost and widespread availability, conventional US is the modality of choice for the initial evaluation of liver resection candidates, and can be used to preclude from surgery patients with diffuse lesions. Following US, in patients eligible for surgery, further CT and/or MRI is usually recommended for surgical planning [5].

Although many series have been published concerning imaging diagnosis in focal liver lesions, mainly with CT and MRI, the optimal imaging staging strategy has not yet

\section{Biomed Central}


been defined, which means that there is no agreement about the best imaging strategy for liver surgical planning.

Intraoperative ultrasound (IOUS) is well established as the best imaging modality for liver lesion depiction and, in some series, is considered the gold standard for liver evaluation [6-8].

The real place for US in liver staging has not been established. Besides the relevant advantages of US, there are drawbacks related to the operator, patient and equipment. As a result, US examination has a wide range of sensitivity rates according to the published series. Low sensitivity rates are usually related to studies performed previous to state-of-the-art equipment and, there are no recent published series focusing on conventional abdominal US exclusively employed for the evaluation of liver nodules before surgery.

This study seeks to determine whether US examination made exclusively for liver evaluation, using modern equipment and operated by experienced radiologists, may contribute to liver staging before hepatic resection. In order to answer this question, the purpose of the study was to evaluate the efficacy of conventional preoperative abdominal US in detecting liver lesions, and the impact of US findings on surgical management.

\section{Methods}

\section{Patients}

From March 2002 to June 2007, 67 patients were studied. After being indicated for curative intent surgery by a multidisciplinary team, patients were sent to the Imaging Department for US liver evaluation.

Patients were included in this study if preoperative US had been performed within 14 days before surgery and cirrhotic patients were excluded. All patients were informed about the study and signed an informed consent.

There were 34 men and 33 women, with a mean age of 57 years (range: 20 to 73 years). Diagnosis included colorectal cancer metastasis $(n=51)$, neuroendocrine tumor metastasis $(n=6)$, peripheral cholangiocarcinoma $(n=3)$, gastrointestinal stromal tumor metastasis $(n=2)$, one melanoma metastasis, one basal cell carcinoma metastasis, one gastric cancer metastasis, one breast cancer metastasis and one bladder cancer metastasis.

\section{Ultrasound}

US examination was performed using ATL HDI-5000 equipment (Philips Medical Systems, Bothel WA, USA) with a convex 2 to $5 \mathrm{mHz}$ probe and, when necessary, a linear 5 to $12 \mathrm{mHz}$ probe and Doppler duplex. US was performed at the dorsal and left lateral decubitus, through an intercostal and subcostal approach with a breath-hold maneuver. Two experienced radiologists, not blind to previous clinical and radiologic data, performed the US and gave the results in consensus.
US findings were recorded by number, size and location of the lesions. Typical hemangioma $(<3 \mathrm{~cm}$ homogeneous hyperechoic and well delimited) and cystic lesions (anechoic lumen, increased through transmission and welldefined wall) were excluded from the analysis. Any lesion not meeting those criteria was included in the analysis.

The number, size and location of liver lesions were also recorded according to the IOUS and, for removed liver segments, histological findings.

The lesion's location was established according to the Brisbane classification: left lateral section (Couinaud's segments II and III), left medial section (Couinaud's segment IV), right anterior section (Couinaud's segments $\mathrm{V}$ and VIII) and right posterior section (Couinaud's segments VI and VII). Any lesion $>40 \mathrm{~mm}$ or occupying more than one of these segments was considered separately.

\section{Surgical plan}

The surgical plan was first established at the multidisciplinary meeting, and, if necessary, modified after the conventional liver US. The definitive surgical strategy was established after palpation and IOUS evaluation. The IOUS was performed by the same radiologist who performed the preoperative US, using Tosbee equipment (Toshiba, Tokyo, Japan) with a T-shaped $7 \mathrm{mHz}$ intraoperative probe bound in sterile plastic.

\section{Data analysis}

Results were evaluated with reference to lesion-by-lesion and patient-by-patient databases and US results were compared with IOUS and histological evaluation results. Histology was considered the gold standard.

Based on the patient-by-patient analysis, surgical planning changes were analyzed, and the total number and mean number of lesions detected per patient by US and by IOUS were compared (by Wilcoxon test and intraclass correlation coefficient).

Based on lesion-by-lesion analysis, the sensitivity, specificity and positive predictive values were calculated for US and IOUS using histological findings as the gold standard. The agreement rate between US and IOUS was calculated by the Kappa test.

Despite not being the main objective of the study, the nonstandardized CT and MRI examinations previously carried out were nevertheless analyzed and the results compared with those of US, since the preoperative surgical decision was based on them. Good quality examinations made within 40 days before surgery were considered for analysis: CT scans with multidetector or spiral equipment with oral and intravenous iodine contrast acquired at precontrast, arterial phase and portal venous phase with a maximum of $5 \mathrm{~mm}$ thickness section were accepted. MRI examinations acquired using high magnetic field equipment with T1-weighted sections, before and after paramagnetic 
contrast administration and dynamic acquisition, with inphase and out-of-phase sequences, and T2-weighted images obtained with and without fat-saturation sequences were accepted.

\section{Results}

\section{Patient-by-patient analysis}

Sixty-seven patients were included in the study. The mean number of lesions detected per patient by US and IOUS was 2.37 and 3.37 , respectively $(P=0.001$; Figure 1$)$. In $52.2 \%$ of patients, US and IOUS depicted the same number of liver lesions. In $4.5 \%$ of patients, US detected more lesions than IOUS; and in $43.3 \%$ of patients, the IOUS depicted from one to seven lesions in addition to the preoperative US results. The intra-class correlation index for US and IOUS was $0.83(P<0.001$; Figure 2$)$.

There was no statistically significant difference in the number of lesions detected by US and by IOUS concerning the presence of steatosis (in US or histology), prior chemotherapy or body mass index.

Surgery with curative intent was conducted in 61 (91\%) patients, including two IOUS-guided radiofrequency ablations. In six patients, intraoperative findings precluded liver resection; one of these patients had melanoma and intraoperative inspection depicted diffuse peritoneal lesions not detected by previous US, CT or MRI. In three patients there were hilar lymph node metastases; in another patient, IOUS accurately identified hepatic vein

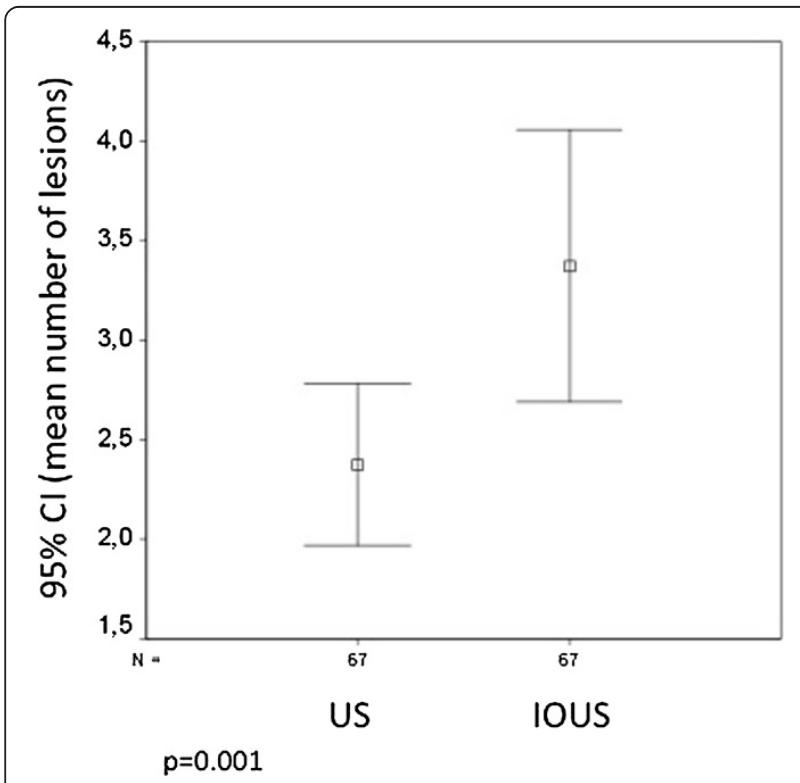

Figure 1 Number of lesions detected per patient by ultrasound and by intraoperative ultrasound. Mean and $95 \%$ confidence interval (CI) for number of lesions detected per patient by ultrasound (US) and by intraoperative ultrasound (IOUS). invasion, precluding resection. Vascular invasion suspicion had already been depicted by the preoperative US. In the last patient, US identified diffuse small liver lesions while CT detected two lesions and positron emission tomography-CT identified only one uptake lesion. Because of these conflicting results, the patient underwent surgical exploration that confirmed preoperative US findings, precluding resection.

The surgical plan, based on previous imaging examinations (CT and/or MRI and US), was modified in 17 (25.5\%) patients after the IOUS. Most of these changes consisted of a new strategy to the previous planned hepatectomy (due to new or more precisely located lesions given by IOUS).

\section{Lesion-by-lesion analysis}

Two-hundred and forty-one lesions were depicted in 67 patients (mean 3.4; range 1 to 13 lesions per patient) with a mean diameter of $24.1 \mathrm{~mm}$ (median $17 \mathrm{~mm}$; range 2 to $120 \mathrm{~mm}$ ).

Histological evaluation was obtained in 196 lesions; 155 were considered malignant (151 metastases and four liver primary tumors) and 41 (20.9\%) benign (Table 1).

Considering the 155 malignant lesions, $24.5 \%$ and $4.5 \%$ were not depicted by US and by IOUS, respectively.

The overall lesion detection rate by US was $65.6 \%$ (158 of $241 ; 95 \%$ confidence interval: $62.2 \%, 74.0 \%$ ), while by nonstandardized CT the rate was $64.8 \%$ (46 of 72 ; $95 \%$ confidence interval: $56.0 \%, 78.0 \%)$ and by nonstandardized

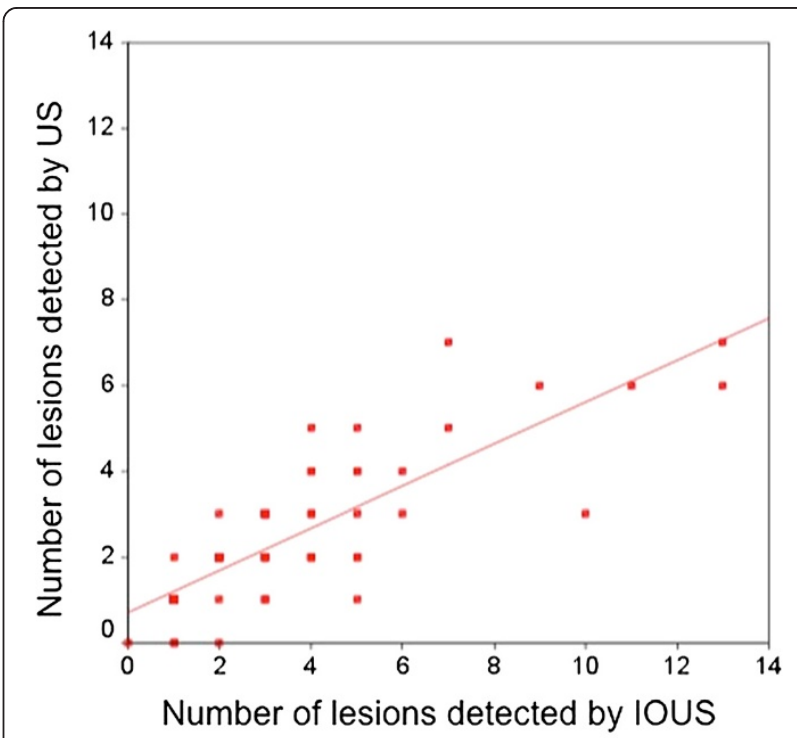

Ricc $=0.83(p<0.001)$

Figure 2 Intra-class correlation coefficient for number of lesions detected per patient by ultrasound and intraoperative ultrasound. Intra-class correlation coefficient (Ricc) for number of lesions detected per patient by ultrasound (US) and by intraoperative ultrasound (IOUS). 


\begin{tabular}{ll}
$\begin{array}{l}\text { Table } \mathbf{1} \text { Number of lesions according } \\
\text { diagnosis }\end{array}$ \\
\hline Histological diagnosis & Number of lesions (\%) \\
\hline Metastases & $151(77.0)$ \\
Cholangiocarcinoma & $3(1.5)$ \\
Hepatocellular carcinoma & $1(0.5)$ \\
Normal liver & $14(7.1)$ \\
Steatosis & $10(5.1)$ \\
Fibrosis / necrosis (post chemotherapy) & $13(5.6)$ \\
Hyperplastic nodule & $3(1.5)$ \\
Hemangioma & $1(0.5)$ \\
Focal nodular hyperplasia & $1(0.5)$ \\
Biliary hematoma & $1(0.5)$ \\
Total & $196(100)$ \\
\hline
\end{tabular}

MRI it was $56.3 \%$ (40 of $71 ; 95 \%$ confidence interval: $59.9 \%, 61.3 \%)$.

Considering 196 lesions submitted to histological evaluation, the sensitivity of US, IOUS, CT and MRI was calculated taking into account the size of the lesions and using histology as the gold standard (Table 2).

The sensitivity rates of imaging methods were lower for lesions $<15 \mathrm{~mm}$, with a statistically significant difference (US: $55.3 \% \times 75.5 \%, P=0.005$; CT: $33.3 \% \times 75.5 \%$, $P=0.013$; MRI: $31.5 \% \times 63.6 \%, P=0.039)$.

Indeed, for lesions $<15 \mathrm{~mm}$, US showed a higher sensitivity rate when compared with the nonstandardized CT and MRI examinations, although this was not statistically significant (US, 55.3\%; CT, 33.3\%; MRI, 31.5\%; $P=0.28$ $(\mathrm{US} \times \mathrm{CT})$ and $P=0.12(\mathrm{US} \times \mathrm{MRI})$ ).

\section{Discussion}

Preoperative imaging techniques play an important role in patient selection and surgical planning for liver resection. According to several authors, US is recommended as the first imaging method for liver lesion detection [9]. For

Table 2 Sensitivity of ultrasound, intraoperative ultrasound, nonstandardized computed tomography and magnetic resonance

\begin{tabular}{llcr}
\hline & \multicolumn{2}{c}{$\begin{array}{c}\text { Sensitivity, \% } \\
\text { (number of lesions) }\end{array}$} & \\
\cline { 2 - 3 } Imaging method & All lesions & Lesions <15 $\mathbf{~ m m ~}$ & $\boldsymbol{P}$ value \\
\hline Ultrasound & $75.5(196)$ & $55.3(96)$ & 0.0050 \\
Intraoperative ultrasound & $95.5(196)$ & $92.3(96)$ & 0.5136 \\
Computed tomography & $75.5(67)$ & $33.3(23)$ & 0.0131 \\
Magnetic resonance imaging & $63.6(55)$ & $31.5(26)$ & 0.0391 \\
\hline
\end{tabular}

Sensitivity using histological evaluation as the gold standard considering separately all lesions and lesions $<15 \mathrm{~mm}$. further surgical planning, CT and MRI are the most frequently employed imaging methods; however, there is no agreement for the best preoperative approach. A multimodality strategy is therefore recommended, since no single modality can accurately detect all liver tumors.

Although limitations related to equipment and operator dependence are drawbacks for US examination, this method yields important advantages due to lack of risks, low cost and availability. Over the last 10 to 15 years, however, there have been few published studies focusing on the efficacy of US on liver lesion detection or on its contribution to liver resection strategy $[5,9]$. Furthermore, published series describing low US sensitivity rates usually rely on the use of nonstate-of-the-art equipment [10].

For more than one-half of the patients (52.2\%) included in this study, the same number of lesions was detected by US and IOUS. Konopke and colleagues presented similar results employing contrast-enhanced US, with 50\% of patients on their series with identical findings for both examinations [11]. These authors achieved lower sensitivity rates for conventional US, comparable with our findings.

Higher sensitivity rates related to conventional US were achieved by Dietrich and colleagues (84.6\%) and Albrecht and colleagues (71\%). These authors used other imaging methods, such as CT and MRI, rather than histology or IOUS as the gold standard, which might have had an impact on sensitivity rates $[12,13]$.

The high sensitivity rate achieved by IOUS (95.5\%) and its higher lesion detection rate per patient when compared with US are expected results according to other studies [6-8]. In the present study, curative intent surgery was achieved in $91 \%$ of patients, a result in accordance with other series, with 87 to $94.1 \%$ curative intent surgery $[14,15]$.

Although not the main objective of this study, nonstandardized CT and MRI were compared with US and IOUS. We did not find any statistically significant difference between the sensitivity rates of these examinations and preoperative US. While US was performed by two experienced radiologists using state-of-the-art equipment, not blinded to previous imaging examinations, CT and MRI scans were performed with nonstandardized protocols or equipment. Indeed, although not ideal, the evaluation of nonstandardized CT and MRI represents the daily practice of a liver surgeon, who receives patients that have already been submitted to radiological evaluation and, based on these results, establishes the surgical plan. For small hepatic lesions ( $<15 \mathrm{~mm})$, US presented higher sensitivity rates (55.3\%) than CT (33.3\%) and MRI (31.5\%), although no significant difference was observed.

Considering that multiple imaging studies are recommended for liver staging, Ward and colleagues suggested that CT and MRI should both be employed routinely on preoperative evaluation [16]. We suggest for 
preoperative evaluation that US could be associated with one section imaging method (CT or MRI) as routine, instead of the association of CT and MRI.

\section{Conclusions}

The relatively high sensitivity rates achieved by US when performed by experienced radiologists and focusing only on liver evaluation, with no significant difference when compared with nonstandardized CT and MRI in the present series, places the method in focus again for new studies, with the aim of lowing costs but not the efficiency, in the routine preoperative staging of liver resection candidates. This study confirms that the best treatment option for a patient with a liver tumor is to be studied by a specialized team and radiologist with focused interest in liver tumors. The imaging method is probably of secondary interest.

\section{Abbreviations \\ CT: Computed tomography; IOUS: Intraoperative ultrasound; MRI: Magnetic resonance imaging; US: Ultrasound.}

\section{Competing interests}

The authors declare that they have no competing interests.

\section{Authors' contributions}

MPC, PH and RC conceived the study and participated in its design and coordination. MPC, MRBP and PNVB participated in acquisition and interpretation of data and helped draft the manuscript. MPC and AGVB performed the literature review and data analysis, and drafted the manuscript. $\mathrm{PH}$ and $\mathrm{RC}$ revised the manuscript. All authors read and approved the final manuscript.

\section{Acknowledgement}

None.

\section{Author details}

1Department of Imaging, AC Camargo Cancer Center, Rua Professor Antônio Prudente, 211, São Paulo, Brazil. 'Faculdade de Medicina, Universidade de São Paulo, Rua Eneas de Aguiar Carvalho, 255, São Paulo, Brazil.

Received: 5 January 2013 Accepted: 1 June 2013

Published: 14 June 2013

\section{References}

1. Tsoulfas G, Pramateftakis MG, Kanellos I: Surgical treatment of hepatic metastases from colorectal cancer. World J Gastrointest Oncol 2011, 3:1-9.

2. Chamberlain RS, Canes D, Brown KT, Saltz L, Jarnagin W, Fong Y, Blumgart LH: Hepatic neuroendocrine metastases: does intervention alter outcomes? J Am Coll Surg 2000, 190:432-445.

3. Di Carlo I: Liver surgery for noncolorectal nonneuroendocrine metastases. HPB 2006, 8:83-84

4. Choti MA, Sitzmann JV, Tiburi MF, Sumetchotimetha W, Rangsin R, Schulick $\mathrm{RD}$, Lillemore KD, Yeo CJ, Cameron JL: Trends in long-term survival following liver resection for hepatic colorectal metastases. Ann Surg 2002, 235:759-766.

5. Braga L, Guller U, Semelka RC: Modern hepatic imaging. Surg Clin North Am 2004, 84:375-400.

6. Soyer P, Elias D, Zeitoun G, Roche A, Levesque M: Surgical treatment of hepatic metastases: impact of intraoperative sonography. AJR Am J Roentgenol 1993, 160:511-514.

7. Zacherl J, Scheuba C, Imhof M, Zacherl M, Langle F, Pokieser P, Wrba F, Wenzl E, Muhlbacher F, Jakesz R, Steininger R: Current value of intraoperative sonography during surgery for hepatic neoplasms. World J Surg 2002, 26:550-554.
8. Cohen MP, Machado MA, Herman P: The impact of intra operative ultrasound in metastases liver surgery. Arq Gastroenterol 2005, 42:206-212.

9. Sietses C, Meijerink MR, Meijer S, van den Tol MP: The impact of intraoperative ultrasonography on the surgical treatment of patients with colorectal liver metastases. Surg Endosc 2010, 24:1917-1922.

10. Wernecke K, Rummeny E, Bongartz G, Vassallo P, Kivelitz D, Wiesmann W, Peters PE, Reers B, Reiser M, Pircher W: Detection of hepatic masses in patients with carcinoma: comparative sensitivities of sonography, $C T$, and MR imaging. AJR Am J Roentgenol 1991, 157:731-739.

11. Konopke R, Kersting S, Bergert H, Bloomenthal A, Gastmeier J, Saeger HD, Bunk A: Contrast-enhanced ultrasonography to detect liver metastases: a prospective trial to compare transcutaneous unenhanced and contrastenhanced ultrasonography in patients undergoing laparotomy. Int J Colorectal Dis 2007, 22:201-207.

12. Dietrich CF, Kratzer W, Strobe D, Danse E, Fessi R, Bunk A, Vossas U, Hauenstein K, Koch W, Blank W, Oudkerk M, Hahn D, Greis C: Assessment of metastatic liver disease in patients with primary extrahepatic tumors by contrast-enhanced sonography versus CT and MRI. World J Gastroenterol 2006, 12:1699-1705.

13. Albrecht T, Blomley MJ, Burns PN, Wilson S, Harvey CJ, Leen E, Claudon M, Calliada F, Correas JM, LaFortune M, Campani R, Hoffmann CW, Cosgrove DO, LeFevre F: Improved detection of hepatic metastases with pulseinversion US during the liver-specific phase of SHU 508A: multicenter study. Radiology 2003, 227:361-370

14. Valls C, Andía E, Sánchez A, Gumà A, Figueras J, Torras J, Serrano T: Hepatic metastases from colorectal cancer: preoperative detection and assesment of resectability with helical CT. Radiology 2002, 218:55-60.

15. Jarnagin WR, Bach AM, Winston CB, Hann LE, Heffernan N, Loumeau T, DeMatteo RP, Fong Y, Blumgart LH: What is the yield of intraoperative ultrasonography during partial hepatectomy for malignant disease? J Am Coll Surg 2001, 192:577-583.

16. Ward J, Robinson PJ, Guthrie JA, Downing S, Wilson D, Lodge JP, Prasad KR, Toogood GJ, Wyatt Jl: Liver metastases in candidates for hepatic resection: comparison of helical CT and gadolinium- and SPIO-enhanced MR imaging. Radiology 2005, 237:170-180.

doi:10.1186/1477-7819-11-138

Cite this article as: Cohen et al:: Focused abdominal ultrasound in preoperative liver surgery staging: a prospective study. World Journal of Surgical Oncology 2013 11:138.

\section{Submit your next manuscript to BioMed Central and take full advantage of:}

- Convenient online submission

- Thorough peer review

- No space constraints or color figure charges

- Immediate publication on acceptance

- Inclusion in PubMed, CAS, Scopus and Google Scholar

- Research which is freely available for redistribution 